Raison Présente (Paris),1997, troisième trimestre, juillet-août-septembre, 101113.

\title{
Problèmes d'éthique et de science
}

\author{
par \\ Michel PATY2
}

Introduction

Les problèmes de comportement et de société reliés à la question de la morale ou de l'éthique et de la science sont avant tout des problèmes pratiques. Mais ils peuvent être éclairés par une mise en place plus profonde, de nature philosophique, d'ailleurs importante parce que cela touche au sens, à la signification de la vie, de nos actes. C'est pourquoi, en vérité, les questions de morale et d'éthique sont d'abord des questions philosophiques. Elles prennent leurs racines dans quelque chose de beaucoup plus fondamental que des comportement immédiats, ou des règles édictées. Elles ne se laissent pas réduire à des questions séparées, qu'il faudrait seulement régler ou décider cas par cas, indépendamment les unes des autres. Cela dit, il se peut que certains problèmes particuliers, de nature pratique, apportent de nouvelles questions générales, comme on le voit avec les questions actuelles relatives à la bioéthique, ou comme on l'a vu naguère avec les questions d'armement atomique et de responsabilité sociale des scientifiques. Je voudrais donc, avant le débat, essayer de situer d'abord philosophiquement, c'est-à-dire selon cette exigence essentielle d'approfondissement, la question des rapports de l'éthique et de la morale avec ce qu'on appelle la science. Puis j'évoquerai, à l'aide de cet éclairage, quelques aspects plus immédiatement actuels et pratiques du problème auquel nous nous trouvons confrontés, dans la réalité sociale de chaque jour.

1. Morale ou éthique?

1 L'origine de ce texte est une contribution (inédite) au Colloque "Quelle place pour la morale ?", organisé par la Ligue de l'Enseignement, le journal La Vie, le Cercle Condorcet, Palais des Expositions, Porte de Versailles, Paris, 15.1.1994 (Atelier "Problèmes de la morale - ou de l'éthique - en science"). La présente version a été remaniée et actualisée.

$2 \quad$ Directeur de recherche au CNRS, Equipe REHSEIS (CNRS) et Université Paris 7Denis Diderot, 2, Place Jussieu, 75251 PARIS-Cedex-05. 
Je commencerai, en premier lieu, par proposer une distinction entre la morale et l'éthique. La morale concerne des règles de comportement individuels et collectifs admis pour une société donnée, par consensus tacite ou explicite, liées aux valeurs admises et au droit reconnu. Elle constitue, en quelque sorte, la norme "transcendante" (c'est-à-dire une loi supérieure s'imposant aux consciences, dans un sens qui a été explicité par Kant) des actions qui relèvent d'une responsabilité individuelle ou sociale. L'éthique porte sur l'attitude de l'être, plus globale et plus profonde que l'obéissance à la règle ou la conformité à des moeurs, expression d'une unité propre de l'être individuel, d'une responsabilité assumée en fonction de valeurs acceptées au plus intime, indépendamment des règles et du droit (voir 1"'homme éthique" de Jean Nabert, et, par exemple, l'oeuvre de Paul Ricoeur).

Les deux, la morale et l'éthique, ne sont pas nécessairement en opposition, mais elles portent, pour ainsi dire, sur des réalités différentes. L'une juge les actes, l'autre s'établit en rapport à une conscience de soi. On ne peut pas, en général, juger de l'extérieur une attitude éthique. Elle représente une exigence interne, dont l'examen peut toutefois nous guider dans nos décisions et dans nos jugements. Ce que l'on peut juger, ce sont les effets apparents, les actes posés, et leur conformité ou non à des valeurs et à des règles, c'est-à-dire ce qui concerne la morale. Cependant, tout le monde est concerné par l'éthique: elle est la marque de ce que toute personne individuelle est une valeur; elle implique la liberté profonde des consciences. Dans les problèmes dont nous allons parler, nous rencontrerons, chacune à sa place et selon les exigences qui lui sont propres, l'éthique et la morale.

\section{Science, pensée, pouvoir}

Considérons maintenant la science, sa réalité, ses dimensions, ses conséquences. Sa réalité comporte plusieurs aspects, que l'on oublie parfois pour s'en tenir aux plus apparents, qui tiennent plutôt à ses effets. Or, la réalité de la science dans le monde contemporain, c'est d'abord qu'elle est une activité qui aboutit à modifier le contenu de la connaissance dont on disposait jusqu'alors. En bref, la science est à la fois un travail de la pensée et un contenu de connaissance (ou une somme de contenus, qu'on appelle le savoir). Mais cette part de sa réalité est inséparable de ses effets, qui très souvent ont nom aujourd'hui technologie et pouvoir.

L'éthique et la morale sont essentiellement intéressées, en ce qui concerne la science, au rapport qui lie la connaissance et le pouvoir et, en premier lieu, à la nature de la connaissance, mais aussi à la nature de ce pouvoir qui tient à elle (ou auquel elle tient). Ce peut être aussi bien le pouvoir qui s'exerce sur la pensée (il en est de bien des façons, qui ne sont pas toutes négatives, et l'on n'oubliera pas que l'ascèse elle-même est un pouvoir: pouvoir sur soi-même). $\mathrm{Ou}$ le pouvoir que l'on exerce sur la nature (celui de la connaître, de la prévoir, de la modifier, de la vivifier ou de la détruire). Mais ce peut être aussi le pouvoir sur les hommes (par exemple, le pouvoir que donne le privilège du savoir, ou l'information, ou même le pouvoir que l'on peut avoir dans l'organisation de la science: pouvoir de juger et de décider sur des choix stratégiques ou sur des 
personnes), le pouvoir dans la société résultant du prestige de celui qui connaît, ou ce pouvoir, plus tangible encore, qui résulte, plus que du savoir, de la fonction acquise grâce à lui et qui peut en être très éloignée, comme on le voit au "mythe de l'expert". Dans la question du pouvoir sur les hommes au nom du savoir, on trouve celle, qui concerne de façon éminente l'éthique, de l'adéquation réelle de ce savoir à la possibilité d'émettre un tel jugement (compétence réelle ou illusoire, mauvaise foi, respect, etc., qui fait référence à l'éthique personnelle de ceux qui ont la fonction de décider, de faire des choix).

\section{La science comme recherche et l'éthique}

En quoi l'attitude devant la connaissance est-elle concernée par l'éthique? Le désir de connaissance a-t-il quelque chose à voir avec l'éthique ? Je crois bien que c'est le cas, et j'invoquerai à l'appui de cette conviction l'apologue du temple de la science proposé par Einstein : l'ange du Seigneur appelle de sa trompette à sortir du Temple de la science où les chercheurs sont rassemblés, ceux qui recherchent en vue des applications ; puis ceux qui le font en vue de la gloire ; puis ceux qui s'y consacrent dans un but idéologique ou apologétique. Il ne reste à la fin qu'un très petit nombre, ceux qui cherchent pour connaître : ils sont justifiés.

Et encore: le choix d'un problème a-t-il quelque chose à voir avec l'éthique ? La réponse est non, sans doute, si l'on s'en tient à un plan purement intellectuel, car l'on admet, dans la plupart des domaines du moins, une neutralité de ces problèmes vis-à-vis des questions éthiques et morales. Le temps n'est pas loin, cependant, où ce n'était pas toujours le cas, et où les problèmes posés dans certaines directions de recherche n'étaient pas neutres idéologiquement, comme les études sur l'eugénisme, dans la première moitié du siècle, liées à des préjugés raciaux. N'excluons pas la possibilité, de nos jours, de recherches proposées comme relevant des sciences humaines et reliées à des objectifs directement intéressés (au profit d'un produit, d'une entreprise, d'une idéologie, d'un Etat). Mentionnons la question des "demandeurs de recherche", en matière de recherche appliquée, dont la gamme est étendue et les implications sont variées, de la préoccupation pour la survie des armées coloniales (qui motiva souvent, par exemple, l'intérêt des grandes puissances pour la recherche bactériologique ou parasitologique) à l'amélioration de la production, mais aussi à celle des conditions de travail et de sécurité (directions de recherches probablement encore trop peu développée dans les cas précis et particuliers, car il s'agit de demandeurs non traditionnels, comme les syndicats de travailleurs ou les mouvements associatifs).

Si l'éthique et la morale ne sont pas toujours - loin s'en faut - concernées par le choix des problèmes, du moins le sont-elles plus souvent au niveau des choix de priorités : cela se marquera, par exemple, par une tendance à favoriser des recherches liées à des résultats - voire à des profits - à court terme, contre des recherches plus fondamentales mais aux résultats incertains, etc.

Une autre dimension est celle du choix d'une position sur une question scientifique et de son rapport éventuel à l'éthique. Il me semble que la conception que l'on se fait de la notion de vérité et l'attitude adoptée à son égard dans la pratique scientifique appartient à cette dimension. Prenons la question du relativisme 
de la connaissance, qui suscite depuis deux décennies engouements et controverses. Certains y voient, non sans quelques raisons, une antidote au scientisme, cette idéologie de la science comme connaissance absolue, intemporelle et neutre par rapport aux débats des hommes et aux vicissitudes de leur histoire sociale, qui sévissait naguère et qui se trouve encore très répandu chez les scientifiques, en raison notamment de la nature de leur formation. La prise en compte, dans l'étude historique, philosophique ou sociale des sciences, des conditions effectives de leur production, qui est effectivement nécessaire mais qui soulève des problèmes délicats de méthodologie, a suscité chez certains l'idée que cette connaissance scientifique dépendrait davantage de facteurs sociaux que de critères objectifs. Pourtant, le fait que la science soit élaborée par des hommes, qu'elle soit construite, que ses acquis soient toujours relatifs et qu'elle soit appelée à des transformations permanentes, ne signifie pas pour autant qu'elle soit une pure construction sociale, dont les contenus seraient d'importance secondaire et pourraient être ignorés ou dissous dans la critique des conditions d'élaboration des énoncés. Dans ses tendances extrèmes, ce relativisme n'est pas autre chose qu'un réductionnisme, aussi simpliste et brutal - et, en définitive, ignorant - que ceux qui ont toujours marqué le scientisme. Le relativisme extrème en matière de connaissance n'est pas autre chose, tout bien considéré, qu'un réductionnisme sociologiste: ce sociologisme prend tout simplement la suite du mécanisme, $d u$ réductionisme physico-chimique, $\mathrm{du}$ biologisme, $\mathrm{du}$ neurologisme ... Au scientisme dur il ne fait qu'opposer un social-scientisme, qui, comme le premier, se veut hégémonique. La différence de stratégie à cet égard n'est qu'apparente. Les scientismes réductionnistes s'arrogent un monopole sur la vérité, identifiée de manière arbitraire à celle, proclamée définitive, de leurs domaines limités de connaissance, et leur méthode est celle de l'autorité. Le sociologisme en matière de connaissance substitue à la notion de rationalité celle d'opinion et à la notion de vérité celle de consensus, faisant, au bout du compte, aussi peu fond sur la liberté et l'amour de l'intelligence. Ses prétentions à l'autorité ne sont pas moindres et, puisqu'il n'est à ses yeux de vérité que celle que l'opinion ratifie, il espère les faire aboutir, avec son ambition, en jouant de ce même registre, le seul qu'il reconnaisse, sur lequel il a projeté l'idée même de connaissance et de sa valeur. Tout laisse à penser, à y bien regarder, que susciter l'engouement et orienter la mode, par les jeux de pouvoir et les effets médiatiques, sont pour lui des moteurs autrement importants que la recherche du vrai.

Les questions qui sont l'objet de controverses de ce genre ne peuvent être considérées comme restreintes à la sphère purement intellectuelle, même si c'est avant tout l'argumentation intellectuelle qui s'impose à leur égard, puisqu'il s'agit de vérité: elles comportent des aspects qui relèvent de l'éthique, et même de la morale. L'idée d'une vérité que nous posséderions, comme, à l'inverse, le renoncement à la notion de vérité, peuvent l'une aussi bien que l'autre relever de raisons non avouables, comme la paresse intellectuelle (l'étroitesse volontaire d'esprit), l'ambition, l'appropriation d'un pouvoir au nom du savoir. Une attitude éthique, à cet égard, n'oblige-t-elle pas à tenter de tenir ensemble les exigences d'amour de la vérité et de doute intellectuel sur la vérité ? Je parle bien de la vérité, parce que l'on doit poser quelque chose, que l'on appelle ainsi, comme ce à quoi la connaissance se propose d'accéder (ou alors tout n'est qu'illusion), le but 
vers lequel on doit tendre dans cette recherche, et l'amour de la vérité est alors le choix délibéré et responsable d'une telle direction comme idéal de dépassement. Et le doute intellectuel sur la vérité (acquise ou supposée), est alors l'attitude qui permet d'aller au-delà des limitations présentes de notre notion de la vérité. Si la notion de vérité telle qu'elle est souvent conçue (associée aux qualificatifs d'absolu, d'intangible, etc.) peut et doit être critiquée, on ne peut supprimer l'idée fondamentale, essentielle, que ce mot exprime, celle d'une exigence qui nous oblige à dépasser l'immédiateté, la facilité, l'apparence, pour respecter "ce qui est", sur lequel l'on dispose d'éléments, ne serait-ce que par la réflexion sur notre propre expérience - à l'aide de notre raison. Nos vérités sont relatives ; du moins, nous savons quelque chose, et nous savons que nous le savons: cela seul nous suffit pour employer le mot de vérité. Reste la question de savoir ce qu'elle est - et c'est tout le problème de la connaissance.

\section{La responsabilité des scientifiques}

La morale et l'éthique ensemble sont concernées par la question de la responsabilité des scientifiques, qui peut se poser ainsi : quel pouvoir les scientifiques, producteurs des connaissances, mais non le plus souvent de leurs effets, peuvent-ils avoir sur l'utilisation de celles-ci ? En vérité, ils ne sont pas les possesseurs de cette connaissance et n'ont pas de pouvoir particulier sur son utilisation parce que les deux sont, dès l'origine, en fait et en droit, du domaine public, res publica. Ils en ont, toutefois, une expérience privilégiée, qui leur confère naturellement un rôle particulier dans le fait qu'elles deviennent véritablement publiques. Ils ont, par là, un devoir d'information, c'est-à-dire celui d'attirer l'attention du public sur ces effets, corrélatif d'un devoir plus général d'éducation et de formation, en suscitant s'il le faut les débats publics que requièrent les démocraties et en y prenant part, comme tout citoyen - car les scientifiques sont avant tout des citoyens, comme les autres, avec les mêmes devoirs et les mêmes droits -, faisant bénéficier la cité de leur expérience et de leurs réflexions, sans autre autorité particulière. En tant que citoyens informés, ils peuvent, en toute légitimité, participer d'un contre-pouvoir au pouvoir des "experts" officiels, lesquels se trouvent bien souvent au service de groupes d'intérêt. La nature de la tâche est alors relativement simple : il s'agit de jeter toute la lumière possible sur des masses d'informations qui circulent dans la cité, souvent contradictoires et données en pâture sans critique avertie, rendant impossible toute décision responsable. Ce devoir de clarification en direction du public est indissociable d'un devoir semblable à l'intérieur du milieu de l'université et de la recherche, par la confrontation libre des idées, le débat, la réflexion et l'étude approfondie faite avec le recul désirable. Le monde de la recherche et de l'université dans son ensemble possède à cet égard une mission qui dépasse la seule production et transmission des connaissances, et qui n'est pas encore suffisamment reconnue: celle de l'étude et de la réflexion sur ces connaissances, sur leurs tenants et leurs aboutissants, selon toutes leurs dimensions, historiques, philosophiques et sociales. 
être seulement le fait de quelques "généralistes de la pensée et de l'action sociale", de "maîtres à penser", comme certains ont pu le suggérer ${ }^{3}$, mais par des scientifiques et d'autres citoyens sans prétention particulière, sinon d'être animés par le vif sentiment de leur responsabilité, et qui surtout ne s'arrogeraient à cet égard aucun monopole. Il ne saurait être question de s'en remettre à de nouveaux gourous - qui seraient d'ailleurs inévitablement imposés par des effets médiatiques - chargés de penser pour les autres, quand c'est d'un débat permanent sur les valeurs humaines, culturelles et sociales que nous avons besoin. Seul un tel débat raisonné, inhérent à l'attitude démocratique, peut habituer chacun à se former l'intuition pratique" qui le guide dans ses comportements en même temps qu'elle permet d'actualiser la morale. Car la morale a besoin d'exercice, et l'on pourrait reprendre à son propos telle maxime de Descartes sur l'exercice de la pensée vraie $^{4}$. On pourrait d'ailleurs penser à un objectif de cette nature pour une action éducative par les medias: entreprendre un examen contradictoire régulier de telles valeurs ou propositions fondatrices de la morale, de l'éthique, de la démocratie, voire de la raison elle-même, qui évolue aussi, en regard de telles connaissances et de tels effets qui en ont résulté.

Un autre aspect de la question de la responsabilité pourrait être également le suivant : quels pouvoir les chercheurs scientifiques ont-ils sur la poursuite de la recherche des connaissances ? J'y reviendrai en terminant.

\section{Science, production, profit, morale}

La science est en même temps production de connaissances et facteur de production, entendant cette dernière dans le sens courant : de cette activité résultent des produits, objets de consommation qui deviennent souvent nécessaires et source de profit. Les découvertes scientifiques représentent des enjeux du point de vue économique, et pas seulement à long terme. Il devient d'ailleurs de plus en plus difficile de séparer à cet égard la recherche fondamentale et la recherche appliquée, la première aboutissant directement, dans certains secteurs, à des "produits" négociables, sans attendre nécessairement le relai de la seconde.

Des exemples quotidiens illustrent cette participation, effective ou possible, de la science à la production et à la vie économique, de la pharmacologie à la biogénétique, de la chimie à la supraconductivité. On a pu craindre récemment que certaines firmes américaines ne se réservent le monopole de la connaissance du génôme humain - si elles y parvenaient avant les autres -, connaissance sur laquelle les journaux titraient: "La conquête du génôme humain devrait donner lieu dans les prochaines années à une fantastique bataille scientifique et commerciale". Et encore: "La compétition scientifique se doublera - aux Etats-Unis notamment - d'une fantastique course aux brevets", pour un marché évalué à 200 milliards de dollars. Quant aux découvertes récentes sur la supraconductivité à température proche de la temérature ambiante, les journaux

3 Voir, par exemple, l'article de Claude Got, Le Monde du 14.9.1993.

$4 \quad$ Descartes, Règles pour la direction de l'esprit. 
indiquaient : "Depuis plusieurs années, les industriels du monde entier attendent ce résultat qui, s'il se confirme, leur ouvrira, demain, de fabuleux marchés".

Dernièrement, les progrès accomplis dans les techniques de clonage, appliquées et réussies semble-t-il, non plus seulement sur des batraciens, mais sur des mammifères comme les brebis et même les vaches 5 , laissent espérer de fructueuses retombées dans l'agro-alimentaire, par la production masive d'animaux d'élevage.

Mis sur le marché, les produits de la recherche scientifique deviennent de simples objets de l'activité mercantile, tout en étant parés de vertus ou de pouvoirs supposés tenir à leur origine dans la recherche et le progrès des connaissances. A la commodité et à l'innovation s'ajoute l'efficacité incontestable qui tient à la connaissance objective et, en dernier ressort, à la vérité. Mais il n'est pas rare que de tels produits soient lancés dans le commerce avant que toutes les garanties soient acquises sur ce point. La possibilité d'un lucre rapide favorise, en l'absence de garde-fous rigoureux comme les autorisations légales, une falsification de produits de cette nature, présentés pour ce qu'ils ne sont pas, ou pas encore.

Tels produits pharmaceutiques supposés guérir l'adénome de la prostate sont l'objet d'informations bien orchestrées qui chantent régulièrement merveilles, démenties peu après: l'enjeu financier est tel que peu chaut, au fond, si les médicaments sont efficaces ou non, ils seront de toutes façons administrés. Cela ne s'apparente-t-il pas, de fait, à la publicité mensongère ? Mais comme le doute demeure et que la possibilité d'améliorations individuelles et occasionnelles reste ouverte, le produit est autorisé et prescrit, et ses tarifs ne laissent aucun doute sur le profit qu'il octroie aux firmes concernées.

Les questions posées par les connaissances médicales, notamment, soulèvent un problème, qui n'est d'ailleurs pas nouveau, mais qui n'avait pas, jusqu'ici, directement à voir avec la science et que celle-ci pare d'un raffinement cruel : l'homme, objet de lucre, non plus par l'appropriation de sa force de travail comme aux beaux jours de l'esclavage ou de l'exploitation du prolétariat (dont on aurait tort, d'ailleurs, de penser qu'ils sont révolus), mais comme personne individuelle ou sujet susceptible de souffrance : avec les technologies biomédicales et pharmaceutiques, le corps et la subjectivité de l'homme sont devenus des sources potentielles de profits fabuleux.

C'est la morale qui est ici concernée: quelles règles déontologiques, quelles lois, protègent et protègeront les êtres humains d'une telle exploitation ? On entrevoit que la solution est avant tout une question de droit de l'homme, de dignité et d'égalité des êtres humains, de leur droit égal à bénéficier des effets de ces connaissances et, en qualité de participants de l'humanité, à bénéficier tout d'abord de ces connaissances mêmes en tant que connaissances, par le canal ou la médiation de la communauté des chercheurs du monde. Ce qui sous-entend que la recherche, comme activité sociale, n'est pas seulement une fonction dans une entreprise, une collectivité ou un Etat, mais une mission publique d'intérêt universel. La récente mise à la disposition des chercheurs du monde entier, gratuitement, de la première carte du génôme humain par l'équipe qui l'a dressée6,

$5 \quad$ Nature, février 1997, New Scientist, 13.3.1997. Voir Le Monde, fin février 1997 et 16-17.3.1997.

$6 \quad$ Le Monde, 17.12.1993. 
constitue à cet égard un préalable qui obligera, espérons-le, à ce qu'il en soit fait de même pour les découvertes à venir.

La situation des pays du Tiers-Monde est particulièrement sensible à ces paspects, et serait directement concernée par de telles dispositions. Il existe, quoiqu'on en ait, des effets de monopole des pays riches sur certains domaines des connaissances, qui contribuent à aggraver la dépendance économique, le retard au développement et l'écart.

On voit que deux problèmes sont par là posés : le premier touche au droit, et son urgence est grande, puisqu'il s'agit de se doter d'une juridiction supranationale, le second concerne la publication des connaissances, opposée au secret ou à la rétention. La solution de ce deuxième problème est elle-même peut-être essentiellement juridique, puisqu'il consiste en la délimitation très exacte de ce qui, par droit d'humanité et d'universalité, doit être communiqué (comme la carte du génôme humain), et de ce qui peut rester objet de brevet, comme les inventions particulières de nature technique, etc. Après tout, des connaissances telles que des théorèmes mathématiques, les lois des mouvements des planètes, celle de la gravitation, la relativité et la physique quantique, aussi bien que la théorie de l'évolution ou les conceptions fondamentales de la biologie moléculaire ont été révélées au monde dès qu'elles ont été trouvées. Bien des connaissances actuelles ou à venir sur la nature ou sur la vie doivent et devront être considérées comme aussi générales et fondamentales. Avec cette particularité, dans le cas qui nous a ici surtout retenus, que c'est de la connaissance de l'homme qu'il est question, c'est-à-dire de tous aussi bien que d'un seul.

Le problème primordial paraît donc bien être de nature juridique. Il importe en premier lieu que la communauté internationale se donne l'arsenal de lois qui protègent la sensibilité et l'intégrité de l'esprit et du corps humain, et que chaque société, chaque Etat, se donne les moyens de faire appliquer ces lois. La question d'une juridiction internationale n'est pas spéciale aux seules connaissances scientifiques, et il existe dans cet ordre des instances compétentes. Il ne s'agit, au fond, que d'élargir le droit international aux aspects évoqués, étant considéré que les attendus devraient en être repris par tout Etat désirant faire partie de la communauté internationale (comme c'est le cas pour bien des règles plus courantes). Ces attendus placeraient en-dehors de tout profit possible les retombées directes de découvertes majeures intéressant l'homme, la vie, la nature, en établissant, par exemple, une liste des connaissances acquises ou susceptibles de l'être, qui ne peuvent être commercialisées, et dont l'information sur elles ne peut faire l'objet de rétention. Les inventeurs, artisans de ces découvertes, ne seraient pas dépossédés pour autant de tout droit, et d'abord de leur droit à la reconnaissance de leur priorité: l'on peut imaginer pour gages de reconnaissance d'autres formes, qui ne soient pas seulement symboliques, que celle des brevets tels qu'ils sont actuellement conçus. La loi supranationale comprendrait évidemment en outre des attendus sur les utilisations possibles de telles découvertes.

Il s'agirait, en somme, d'instituer un droit de regard mondial, qui ne serait que l'expression d'un aspect de la loi morale universellement admise, concernant la conscience de l'humanité entière sur elle-même et comprenant son droit imprescriptible sur des découvertes scientifiques ayant des effets potentiels sur 
elle. On comptera, évidemment, avec les problèmes que posent l'édiction d'un tel droit supranational et la nécessité de le faire respecter. Ces problèmes sont de même nature que ceux posés par le contrôle des armements nucléaires ou le respect des droits de l'homme, et comportent toutes les ambiguïtés que nous connaissons dues aux rapports de force actuels entre les Etats. On soulignera toutefois, dans le cas qui nous concerne, une caractéristique originale, susceptible de dépasser ces ambiguités, puisque ce seraient les Etats riches - riches, ici, de leurs recherches - qui seraient les principaux obligés : leur acceptation de se soumettre au contrôle de la juridiction internationale serait un test de leur bonne foi dans leur profession d'égale appartenance à la communauté des nations ${ }^{7}$.

\section{Liberté de la recherche et morale}

Toutes ces considérations plaident en même temps pour la liberté de la recherche fondamentale. Une limitation de son objet au nom d'une autorité quelconque aurait, comme l'histoire des sciences nous l'enseigne, des effets imprévisibles sur les possibilités futures. L'idée d'un moratoire pour ralentir ou arrêter momentanément des recherches génétiques qui peuvent avoir immédiatement des retombées dangereuses, porte, me semble-t-il, le risque d'interdire en même temps des connaissances bénéfiques imprévisibles (par exemple liées à la guérison de la myopathie, ou du SIDA).

Cela ne signifie pas pour autant que tout soit permis. La question que l'on doit se poser me paraît être celle-ci : qu'est-ce qui, dans la recherche, s'oppose à la morale ou à l'éthique ? Les futures connaissances imprévisibles ? certainement pas, puisque nous ne pouvons rien en dire a priori, et répondre ainsi ne serait qu'un autre nom donné à la crainte de la connaissance. Mais, par contre, le non-respect des valeurs fondamentales (telles que la dignité humaine, l'intégrité des dispositions vitales de la nature, etc.) en relation à l'objet de recherche choisi, ou plutôt à la méthode employée à cet égard, est à coup sûr contraire à l'éthique et à la morale. Si, par exemple, au lieu de disséquer les cadavres, l'on disséquait les vivants (et l'on sait que les expériences sur les organes peuvent présenter des cas spécifiques de cet ordre ${ }^{8}$ ). Ou si telle recherche physique, chimique ou biologique avait comme effet nécessaire une contamination irréversible et incontrôlable de la nature. Ou, d'une manière générale, toute recherche à risque qui serait effectuée en l'absence de conditions assurées et rigoureuses de contrôle: le respect de telles conditions apparaît comme la contrepartie que les scientifiques doivent à la société en échange de la liberté de la recherche qu'elle-même leur doit.

Ces devoirs réciproques, dictés par la morale et l'éthique, concernent l'humanité entière, dans l'ordre de la connaissance et dans celui de la sécurité, et

7

Il va de soi que la juridiction supranationale ne serait qu'en partie l'émanation des

Etats.

8 L'opinion mondiale réprouve spontanément les actes de cette nature, commis par divers Etats, tels que l'utilisation de malades mentaux, de condamnés, de prisonniers ou de soldats comme cobayes pour des expériences chimiques ou nucléaires, révélés récemment au public. On connait aussi de nombreux cas de transplantation d'organes au détriment de pauvres dans certains pays du Tiers-Monde. 
ils relèvent eux aussi d'un droit à établir, ou à développer. 